\title{
Dopant Introduced Valley Polarization, Spin, and Valley Hall Conductivity in Doped Monolayer $\mathrm{MoS}_{2}$
}

\author{
Sintayehu Mekonnen (iD) and Pooran Singh \\ Addis Ababa University, Department of Physics, Addis Ababa, Ethiopia \\ Correspondence should be addressed to Sintayehu Mekonnen; hailemariamsintayeh@gmail.com
}

Received 7 February 2018; Revised 27 April 2018; Accepted 2 May 2018; Published 1 August 2018

Academic Editor: Bakhtiar U. Haq

Copyright (C) 2018 Sintayehu Mekonnen and Pooran Singh. This is an open access article distributed under the Creative Commons Attribution License, which permits unrestricted use, distribution, and reproduction in any medium, provided the original work is properly cited.

\begin{abstract}
We study valley polarization, spin, and valley Hall conductivity in doped monolayer $\mathrm{MoS}_{2}$ considering dopant introduced magnetic exchange field using low energy effective Hamiltonian. We found that dopant introduced magnetic exchange field breaks the time inversion symmetry and decouples the energetically degenerated valleys into nondegenerate. Moreover, the calculated result reveals that, at low temperature, in insulating regime, anomalous Hall conductivity in a single valley and the total valley Hall conductivity are quantized, whereas the total spin Hall conductivity vanishes identically. We also found that the strength of the spin-orbit coupling together with the exchange field determines the valley polarization, which in turn controls valley and spin Hall conductivity in doped monolayer $\mathrm{MoS}_{2}$ system. The spin Hall and valley Hall conductivity is dissipationless in the absence of any external magnetic field. Therefore, our results are crucial to generate low power electronics devices.
\end{abstract}

\section{Introduction}

The charge and spin degrees of freedom (DOF) of electrons are at heart of modern electronics as they form the basis of a wide range of applications, such as transistors, photodetectors, and magnetic memory devices. In addition to this, electrons in two-dimensional (2D) crystals that have a honeycomb lattice structure possess an extra valley DOF in addition to charge and spin [1]. Valley is a quantum number defined in an electronic system whose band structure contains energetically degenerate but nonequivalent structures due to a certain crystal structure. Among various material candidates for valleytronics, spatial inversion symmetry broken two-dimensional (2D) honeycomb lattice systems such as graphene and monolayer $\mathrm{MoS}_{2}$ are predicted to be the most useful. These systems have two valleys called $\mathrm{K}$ and K'. The use of valley indexes for a potential information carrier was first suggested in the studies of conventional semiconductors such as AlAs and $\mathrm{Si}$ [2].On the other hand, the presence of a valley-dependent orbital magnetic moment in graphene with gap suggests that currents flow perpendicular to applied electric field even in the absence of a magnetic field, named the 'valley Hall effect.' The valley Hall effect was first observed in graphene with substrate. However, due to the small value of the energy band gap this effect has not been yet observed experimentally. Recently, photogenerated valley Hall conductivity was observed in monolayer $\mathrm{MoS}_{2}$ [3]. In contrast, to graphene in monolayer $\mathrm{MoS}_{2}$ the inversion symmetry is explicitly broken [2], which can give rise to the valley Hall effect where carriers in different valleys flow to opposite transverse edges when an in-plane electric field is applied $[4,5]$. In addition to this, $\mathrm{MoS}_{2}$ has a strong spinorbit coupling (SOC) originating from the $\mathrm{d}$ orbitals of the heavy metal atoms(Mo) [4] and can be an interesting platform to explore spin physics and spintronics applications which are absent in graphene due to its vanishing SOC $[6,7]$. However, as far as both valleys are concerned due to time reversal symmetry analogy to paramagnetic and paraelectric materials, pure monolayers $\mathrm{MoS}_{2}$ are paravlley material. Therefore, the major challenge in valleytronics is to break the degeneracy between the two prominent $\mathrm{K}^{+}$and $\mathrm{K}^{-}$valleys so as to achieve the valley polarization. Recent experimental investigation demonstrated optical means of breaking valley degeneracy [8-11]. In addition to this, other approaches like 
applied electric field[12], by electron-electron interaction[13], by means of external magnetic field [14-17], are also recently proposed mechanism to break valley degeneracy.

In this paper, we theoretically study dynamics of valley polarization, spin, and valley Hall conductivity in the vicinity of $\mathrm{K}^{+}$and $\mathrm{K}^{-}$valleys of $2 \mathrm{D}$ hexagonal Brillouin zone (BZ) of monolayer $\mathrm{MoS}_{2}$ considering spin-orbital coupling and dopant introduced exchange field. In second section using model Hamiltonian together with Berry phase approach we obtained explicit expression of Berry curvature, spin and valley split form of Hall conductivity, and total valley and spin Hall conductivity. In section three, we present the numerical results showing effect of the spin-orbit coupling and dopant introduced exchange field on energy band structure of two prominent valleys $\left(\mathrm{K}^{+}\right.$and $\mathrm{K}^{-}$) and total valley and spin Hall conductivity. Finally the conclusion is presented in last section.

\section{Theoretical Model}

We consider an effective three-band tight binding model in the vicinity of $\mathrm{K}^{+}$and $\mathrm{K}^{-}$valleys of hexagonal $\mathrm{MoS}_{2}$ Brillouin zone including spin-orbital coupling originating from transition element Mo 4d states and the dopant introduced Hamiltonian. In symmetry based three-band tight binding model, the conduction bands are dominated by $4 d z^{2}$ and the valence bands are dominated by $4 d_{x y}$ and $4 d x^{2}-y^{2}$. Since a three-band TB model is enough to capture the bandedge properties in the vicinity of $\mathrm{K}^{ \pm}$valleys [15-17] including energy dispersions, Berry curvature, and valley Hall and spin Hall conductivity. Therefore, it is convenient to use this model to study desired properties in the vicinity of those valleys.

The Hamiltonian of the system will have the form

$$
\widehat{H}=\widehat{H}_{k \cdot p}+\widehat{H}_{S o}+\widehat{H}_{e x c}^{d o p} .
$$

Following $[2,18]$ the low energy effective Hamiltonian in K.P theory of the system reads

$$
\widehat{H}_{k . p}=a t\left(\nu \tau_{x} k_{x}+\tau_{y} k_{y}\right)+\Delta_{m} \tau_{3}
$$

and spin-orbital term Hamiltonian can be written as

$$
\widehat{H}_{s o}=\nu \lambda_{s o}\left(1-\tau_{3}\right) s_{z}
$$

whereas exchange energy resulting from dopants is assumed to be

$$
\widehat{H}_{e x}^{d o p}=-v s_{z} h_{e x} \sigma_{z}
$$

Here, $\widehat{H}_{\text {exc }}^{\text {dop }}$ is magnetic dopant $(\mathrm{Mn}, \mathrm{V})$ induced magnetic exchange energy and is the energy splitting resulting from exchange interaction between dopants, $t$ and a are firstorder valence (conduction) band effective hopping and lattice constant for $\mathrm{MoS}_{2}$, respectively, and $\Delta_{m}$ and $\lambda_{\text {so }}$ are mass correction for Dirac Hamiltonian and spin-orbit coupling parameter, respectively. $v= \pm 1$ designates the value index for $K^{ \pm}, \tau_{x, y, z}$ and $\sigma_{x, y, z}$ are Pauli matrices in lattice space and spin space, respectively. Using the value of Dirac matrices the matrix form of Hamiltonian reads

$$
\widehat{H}(k)=\left(\begin{array}{cc}
\Delta_{m}-v s_{z} h_{e x} & a t k_{-}^{\nu} \\
a t k_{+}^{\nu} & \nu s_{z}\left(h_{e x}-\lambda_{s o}\right)-\Delta_{m}
\end{array}\right),
$$

where we have introduced short notation $k_{-}^{\nu}=k_{x} v-i k_{y}$ and its complex conjugates $k_{+}^{v}=k_{x} v+i k_{y}$. The eigenfunction of tight binding Hamiltonian can be expressed in terms of product of periodic part and plane wave as

$$
\psi_{p}(k)=\frac{1}{\sqrt{A}} \exp (i k . r) u_{p}^{v}(k)
$$

where $\mathrm{A}$ is area of the two-dimensional system and $(1 / \sqrt{A}) \exp (i k . r)$ is the plane wave solution corresponding to kinetic (hopping term) of Hamiltonian in (5) and $u_{s}^{v}(k)$ is periodic part of Bloch state which can be written as a two-component way considering spin-up and spin-down components. After solving characteristic equation, the energy eigen value and energy eigenvector, respectively, is obtained as

$$
\begin{aligned}
\zeta_{p}^{v}(k)= & -v s_{z} \frac{1}{2} \lambda_{s o} \\
& +p \sqrt{\left(\Delta_{m}+v s_{z}\left(\frac{1}{2} \lambda_{s o}-h_{e x}\right)\right)^{2}+a^{2} t^{2} k^{2}} . \\
\psi_{p}^{v}(k)= & \frac{\exp (i k \cdot r)}{W}\left(\begin{array}{c}
a t\left(v k_{x}-i_{k y}\right) \\
\zeta_{p}^{v}(k)+v s_{z}\left(h_{e x}-\Delta_{m}\right)
\end{array}\right),
\end{aligned}
$$

where

$W$

$$
\begin{aligned}
& =\sqrt{\left(v s_{z}\left(h_{e x}-\frac{1}{2} \lambda_{s o}\right)-\Delta_{m}+p D_{s_{z}}^{v}(k)\right)^{2}+a^{2} t^{2} k^{2} .} \\
& D_{s_{z}}^{v}(k)=\sqrt{\left(\Delta_{m}+v s_{z}\left(\frac{1}{2} \lambda_{s o}-v h_{e x}\right)\right)^{2}+a^{2} t^{2} k^{2} .}
\end{aligned}
$$

On the other hand, from our previous work [19], the general expression for $\mathrm{z}$ component Berry curvature which is analogical with real space magnetic field is given by expression

$$
\vec{\Omega}_{x, y}^{n}(z)=-2 \operatorname{Im} \sum_{m \neq n} \frac{\left\langle u_{n}(k) \mid \nabla_{\alpha} \widehat{H}(k) u_{m}(k)\right\rangle\left\langle u_{m}(k) \mid \nabla_{\beta} \widehat{H}(k) u_{n}(k)\right\rangle}{\left(\varepsilon_{m k}-\varepsilon_{n k}\right)^{2}} e_{z} .
$$


Now, fascinatingly, this expression of Berry curvature for particular system is under consideration together with using (7) and (8). Making transformation, $\epsilon_{n k}-\epsilon_{m k} \longrightarrow \zeta_{+}^{v}(k)-$ $\zeta_{-}^{v}(k), \nabla_{x} \longrightarrow \partial / \partial k x$, and $\nabla_{y} \longrightarrow \partial / \partial k y$ using those variable changes in (11), we shall rewrite Berry curvature of conduction band minimum (CBM) and valance band maximum (VBM) in the vicinity of $\mathrm{K}^{-}$and $\mathrm{K}^{+}$valleys as

$$
\Omega_{x, y}^{v}(z)=-2 \operatorname{Im} \frac{\left\langle u_{+}^{v}(k) \mid(\partial / \partial k x)(\widehat{H}(k)) u_{-}^{v}(k)\right\rangle\left\langle u_{-}^{v}(k) \mid(\partial / \partial k y)(\widehat{H}(k)) u_{+}^{\nu}(k)\right\rangle}{\left(\zeta_{+}^{v}(k)-\zeta_{-}^{v}(k)\right)^{2}} e_{z}
$$

where $\mathrm{x}$ and $\mathrm{y}$ component of velocity in (12) can be obtained using the relation between wave vector dependent Hamiltonian in (5) and momentum operator as

$$
\begin{aligned}
& \widehat{v}_{x}=\frac{\partial \widehat{H}(k)}{\hbar \partial \widehat{k}}=v \frac{1}{\hbar} a t \widehat{\tau}_{x}, \\
& \widehat{v}_{y}=\frac{\partial \widehat{H}(k)}{\hbar \partial \widehat{k}}=\frac{1}{\hbar} a t \widehat{\tau}_{y} .
\end{aligned}
$$

Substituting (13) and (14) into (12) together with simple manipulation yields the Berry curvature for upper and lower band $( \pm)$ as

$$
\Omega_{x, y}^{ \pm}=\mp \frac{v a^{2} t^{2}}{\hbar^{2} D_{s_{z} v}^{2}} e_{z}
$$

In (15), $e_{z}$ is unit vector along $\mathrm{Z}$ axis. To obtain the expression $e_{z}$ explicitly we introduce a three-dimensional unit vector along $\mathrm{x}, \mathrm{y}$, and $\mathrm{z}$ in a two-dimensional plane in $\mathrm{k}$ space in terms of eigenvalues as

$$
\begin{aligned}
& \widehat{n}(k) \\
& \quad=\left\{\frac{v a t k_{x}}{D_{s_{z}}^{v}(k)}, \frac{a t k_{y}}{D_{s_{z}}^{v}(k)}, \frac{\Delta_{m}+s_{z} \nu\left((1 / 2) \lambda_{s o}-h_{e x}\right)}{D_{s_{z}}^{v}(k)}\right\} .
\end{aligned}
$$

Using (16) into (15), we find

$$
\Omega_{v}^{p}(z)=-p v \frac{a^{2} t^{2}\left(\Delta_{m}+v s_{z}\left((1 / 2) \lambda_{s o}-h_{e x}\right)\right.}{2 D_{s_{z}}^{v}(k)^{3}}
$$

where $p=1$ for conduction band curvature and $p=-1$ for valence band curvature. On the other hand, using linear response theory the quantum Kubbo formula for Hall conductivity in current-Current correlated system is found to be

$$
\begin{aligned}
& \left.\sigma_{\mu \nu}(q, \omega)\right)=\frac{i \hbar}{V^{d}} \\
& \cdot \sum_{n, m}\left\langle\frac{f\left(E_{n}\right)-f\left(E_{m}\right)}{\left(E_{n}-E_{m}\right)\left(E_{n}-E_{m}+\hbar(\omega+i \eta)\right.} J_{\mu}^{n m}(q)\right. \\
& \left.\cdot J_{v}^{m n}(-q)\right\rangle_{c} .
\end{aligned}
$$

In dilute limit or in absence of disorder effect, after some mathematical algebra, $\mathrm{z}$ components of conductivity in (18) are simplified to

$$
\begin{aligned}
& \left.\sigma_{x, y}^{\nu}(z)=\frac{q^{2}}{\hbar V^{d} \sum_{k}\left(f_{\zeta_{-}^{v}(k)}-f_{\zeta_{+}^{v}(k)}\right.}\right) . \\
& \quad\left(\operatorname{Im} \sum_{+\neq-} \frac{\left\langle u_{+k}^{v} \mid \nabla_{x} \widehat{H}(k) u_{k-}^{v}\right\rangle\left\langle u_{-, k}^{v} \mid \nabla_{y} \widehat{H}(k) u_{+k}^{v}\right\rangle}{\left(\zeta_{-}^{v}(k)-\zeta_{+}^{v}(k)\right)^{2}}\right) \\
& \quad \cdot e_{z}
\end{aligned}
$$

On account of (12), the right side of (19) inside the bracket gives $\left(-\Omega^{p}(z) / 2\right)$ which reproduces conductivity in terms of Berry curvature as

$$
\sigma_{\nu}^{p}(z)=-\frac{q^{2}}{2 \hbar V} \sum_{k}\left(f_{\left(\zeta_{k_{-}}\right)}-f_{\left(\zeta_{k_{+}}\right)}\right) \Omega_{\nu}^{p}(z)
$$

Following $[20,21] \mathrm{z}$ component of total spin and valley Hall conductivity in the vicinity of both Valleys, namely, $\mathrm{K}^{+}$and $\mathrm{K}^{-}$, respectively, can be obtained using

$$
\begin{aligned}
\sigma^{\text {spin }}(z) & =\left(\left(\sigma_{\uparrow}^{+}(z)-\sigma_{\downarrow}^{+}(z)\right)+\left(\left(\sigma_{\uparrow}^{-}(z)-\sigma_{\downarrow}^{-}(z)\right) .\right.\right. \\
\sigma^{\text {val }}(z) & =\left(\left(\sigma_{\uparrow}^{+}(z)+\sigma_{\downarrow}^{+}(z)\right)-\left(\left(\sigma_{\uparrow}^{-}(z)+\sigma_{\downarrow}^{-}(z)\right) .\right.\right.
\end{aligned}
$$

where \pm designates short notation for $\mathrm{K}^{+}$and $\mathrm{K}^{-}$valleys, respectively.

First we evaluate both spin and valley split form of anomalous Hall conductivity $\sigma_{\uparrow, \downarrow}^{ \pm}$using (20) but considering two limiting cases.

Case I. At absolute zero and at low impurity concentration or in pure monolayer $\mathrm{MoS}_{2}$, in this scenario all electron states below the Fermi level will contribute (i.e., it is a Fermi sea property). We use the approximation $f\left(\zeta_{k+}\right)=0$ and $f\left(\zeta_{k-}\right)=1$. Here it should be known that the word 'anomalous' reflects Hall conductivity in absence of external magnetic field. Therefore, in this limit after transforming summation into integration, (20) becomes

$$
\begin{aligned}
& \sigma_{v}^{p}(z)=\frac{p v q^{2}}{2 h}\left(\Delta_{m}+v s_{z}\left(\frac{1}{2} \lambda_{s o}-h_{e x}\right)\right) \\
& \cdot \int_{0}^{\infty} \frac{a^{2} t^{2} k d k}{2\left(\left(\Delta_{m}+v s_{z}\left((1 / 2) v \lambda_{s o}-h_{e x}\right)\right)^{2}+a^{2} t^{2} k^{2}\right)^{3 / 2}}
\end{aligned}
$$


After solving (23) the anomalous Hall conductivity in Valley $\mathrm{K}^{+}$in occupied valance band, for spin-up and spin-down electrons (holes)

$$
\Longrightarrow \sigma_{\uparrow}^{+}(z)=\sigma_{\downarrow}^{+}(z)=-\frac{q^{2}}{h} .
$$

Similar to anomalous Hall conductivity in the vicinity of $K^{-}$ valleys in occupied valance band, for spin-up and spin-down electrons (holes),

$$
\sigma_{\uparrow}^{-}(z)=\sigma_{\downarrow}^{-}(z)=\frac{q^{2}}{h} .
$$

From these results we can understand that, in pure limit, anomalous Hall conductivity in a single spin state (spin-up or spin-down) and in single valley $( \pm)$ is quantized (depends only on fundamental unit of conductivity). On the other hand, as seen from (24) and (25) the signs of calculated anomalous Hall conductivity for $\mathrm{K}^{+}$and $\mathrm{K}^{-}$are opposite to each other which indicates that those valleys are related to time reversal symmetry.

The total spin Hall conductivity and valley Hall conductivity in the vicinity of both valleys $\left(\mathrm{K}^{+}\right.$and $\left.\mathrm{K}^{-}\right)$is obtained by substituting (24) and (25) into (21) and (22)

$$
\begin{gathered}
\sigma^{\text {spin }}(z)=0 . \\
\sigma^{\text {valley }}(z)=-4 \frac{q^{2}}{h} .
\end{gathered}
$$

Case II. At finite doping concentration, in this limit both spin hall and valley Hall conductivity should be calculated by taking into consideration both occupied bands from $-\infty$ to 0 plus the conduction band until $\left(\epsilon_{F}\right)$ or $\left(k_{F}\right.$. Hence in this limit (20) is rewritten as

$$
\begin{aligned}
\sigma_{A H C}^{p}(z)= & \frac{q^{2}}{2 \hbar V} \sum_{-\infty<k<0}\left(f_{\left(\zeta_{k_{+}}\right)} \Omega_{\nu S_{z}}^{p}(z)\right. \\
& -\sum_{0<K_{F}}\left(f_{\left(\zeta_{k_{-}}\right)}^{p} \nu S_{z}(z) .\right.
\end{aligned}
$$

After transforming summation into integration

$$
\sigma_{A H C}^{p}(z)=\frac{q^{2}}{2 h} \int_{-\infty}^{0} \Omega_{\nu S_{z}}^{p}(z)-\int_{0}^{k_{F}} \Omega_{\nu S_{z}}^{p}(z)
$$

Substitute (17) into (29) and evaluating for $\sigma_{\uparrow}^{+}(z), \sigma_{\downarrow}^{+}(z)$, $\sigma_{\uparrow}^{-}(z)$, and $\sigma_{\downarrow}^{-}(z)$ by varying the values of $\nu$ and $s_{z}$ in (20). To evaluate $\sigma_{\uparrow}^{+}$from (20), we use $v=1, s_{z}=1, \mathrm{p}=-1$ and after series of steps

$$
\begin{aligned}
& \Longrightarrow \sigma_{\uparrow}^{+}(z) \\
& =-\frac{q^{2}}{h} \frac{\Delta_{m}+(1 / 2) \lambda_{s o}-h_{e x}}{\sqrt{\left(\Delta_{m}+(1 / 2) \lambda_{s o}-h_{e x}\right)^{2}+a^{2} t^{2} k_{F}^{2}}} .
\end{aligned}
$$

Similarly substitution of $v=1, s_{z}=-1$, results in the expression of spin-down electrons in the vicinity of $K^{+}$valleys as

$$
\begin{aligned}
& \Longrightarrow \sigma_{\downarrow}^{+}(z) \\
& =-\frac{q^{2}}{h} \frac{\left(\Delta_{m}+h_{e x}-(1 / 2) \lambda_{s o}\right)}{\sqrt{\left(\Delta_{m}+h_{e x}-(1 / 2) \lambda_{s o}\right)^{2}+a^{2} t^{2} k_{F}^{2}}}
\end{aligned}
$$

Following the same procedure, $\sigma_{\uparrow}^{-}(z)$ and $\sigma_{\downarrow}^{-}(z)$ are found to be

$$
\begin{aligned}
& \sigma_{\uparrow}^{-}(z)=\frac{q^{2}}{h} \frac{\left(\Delta_{m}+h_{e x}-(1 / 2) \lambda_{s o}\right)}{\sqrt{\left(\Delta_{m}+h_{e x}-(1 / 2) \lambda_{s o}\right)^{2}+a^{2} t^{2} k_{F}^{2}}} . \\
& \sigma_{\downarrow}^{-}(z)=\frac{q^{2}}{h} \frac{\left(\Delta_{m}+(1 / 2) \lambda_{s o}-h_{e x}\right)}{\sqrt{\left(\Delta_{m}+(1 / 2) \lambda_{s o}-h_{e x}\right)^{2}+a^{2} t^{2} k_{F}^{2}}} .
\end{aligned}
$$

Total Spin Hall conductivity at finite doping concentration at zero temperature can be obtained by substituting ((30)-(33)) into (21) as

$$
\begin{aligned}
\sigma^{\text {spin }}(z) & \frac{2 q^{2}}{h}\left(\frac{\left(\Delta_{m}+h_{e x}-(1 / 2) \lambda_{s o}\right)}{\sqrt{\left(\Delta_{m}+h_{e x}-(1 / 2) \lambda_{s o}\right)^{2}+a^{2} t^{2} k_{F}^{2}}}\right. \\
& \left.-\frac{\Delta_{m}+(1 / 2) \lambda_{s o}-h_{e x}}{\sqrt{\left(\Delta_{m}+(1 / 2) \lambda_{s o}-h_{e x}\right)^{2}+a^{2} t^{2} k_{F}^{2}}}\right) .
\end{aligned}
$$

Finally, the total valley Hall conductivity can be calculated by making use of those ((30)-(33)) into (22)

$$
\begin{aligned}
& \sigma^{v a l}(z) \\
& =-\frac{2 q^{2}}{h}\left(\frac{\left(\Delta_{m}+h_{e x}-(1 / 2) \lambda_{s o}\right)}{\sqrt{\left(\Delta_{m}+h_{e x}-(1 / 2) \lambda_{s o}\right)^{2}+a^{2} t^{2} k_{F}^{2}}}\right. \\
& \left.+\frac{\Delta_{m}+(1 / 2) \lambda_{s o}-h_{e x}}{\sqrt{\left(\Delta_{m}+(1 / 2) \lambda_{s o}-h_{e x}\right)^{2}+a^{2} t^{2} k_{F}^{2}}}\right) .
\end{aligned}
$$

Equation (34) reveals that unlike the case of pure, limit the total spin Hall conductivity is finite due to spin polarization of $\mathrm{K}^{-}$and $\mathrm{K}^{+}$valleys associated with dopant introduced magnetic exchange field $\left(\mathrm{h}_{e x}\right)$.

\section{Result and Discussion}

The energy plotted as a function of dimensionless wave number $(k a / \pi)$ is displayed in Figure 1. In Figures 1(a) and (b), we find that for zero spin-orbital coupling $(l o=0)$ and zero dopant introduced exchange field $\left(h_{e x}=0\right)$, both bands $\left(\mathrm{K}^{+}\right.$and $\mathrm{K}^{-}$) valleys are degenerate. On the other hand, 


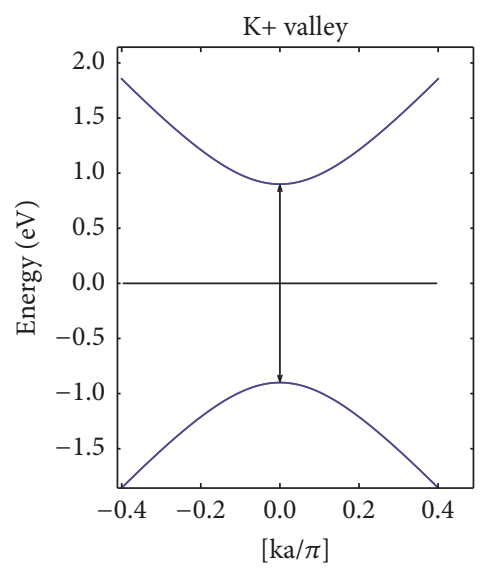

up state, $\mathrm{hex}=\mathrm{lo}=0$
down

(a)

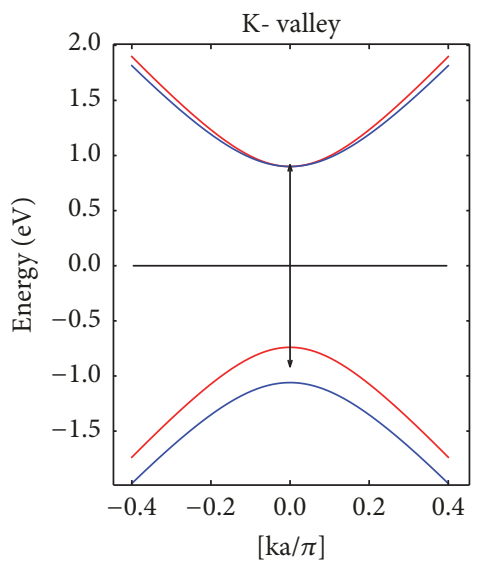

- up hex $=0, \mathrm{lo}=08 \mathrm{eV}$

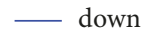

(d)

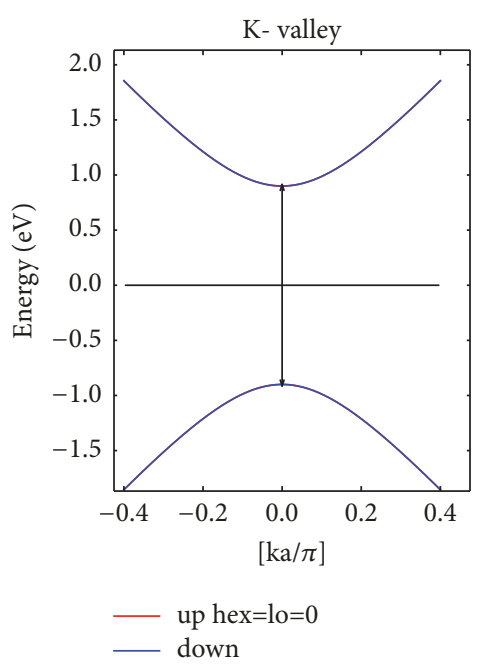

(b)

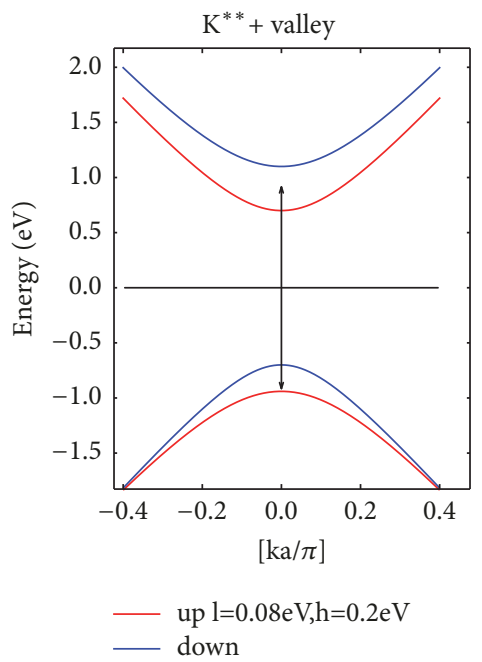

(e)

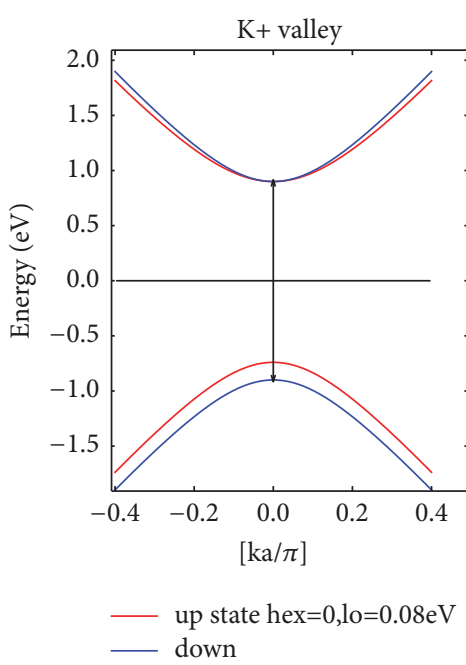

(c)

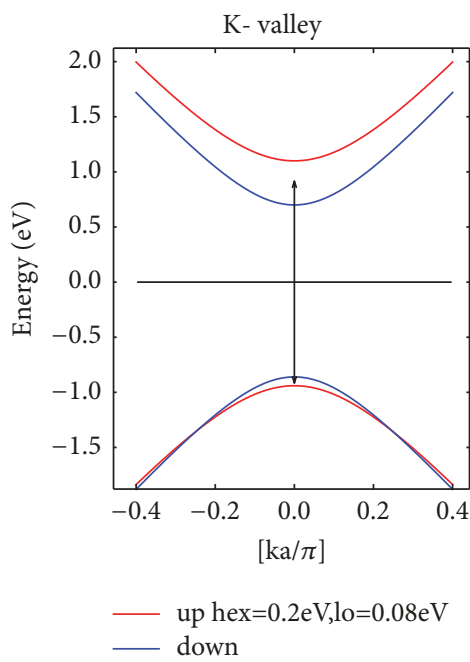

(f)

FIGURE 1: Electronic band structure near valley $K^{ \pm}$: (a) and (b) for zero both spin-orbit coupling and exchange field (lo=hex=0), (c) and (d) for spin-orbit coupling $(\mathrm{lo}=0.08 \mathrm{eV}$,) and zero exchange field, and (e) and (f) for spin-orbit coupling $(\mathrm{lo}=0.08 \mathrm{eV})$ and exchange field (hex $=0.2$ $\mathrm{eV}$ ). Red: spin-up electron (hole) states, blue: spi- down electron (hole) states, right panel for $\mathrm{K}^{+}$, and left panel for $K^{-}$valley.

for nonzero spin-orbit coupling and zero exchange field as shown in Figures $1(\mathrm{c})$ and $1(\mathrm{~d})$, conduction band $\mathrm{K}^{+}$point eigenvalues are identical to those of the $\mathrm{K}^{-}$point if spinup and down are exchanged but the degeneracy in valence band hole state in $\mathrm{K}^{+}$and $\mathrm{K}^{-}$valleys are lifted due to spinorbital coupling resulting from transition metal(Mo) orbitals. However,for finite spin-orbit coupling(lo) and finite dopant introduced exchange field degeneracy in both valence band holes and conduction band electrons in valley $\mathrm{K}^{+}$and $\mathrm{K}^{-}$ is lifted Figures 1(e) and 1(f). Moreover, to understand the role of dopant introduced magnetic exchange field $\left(\mathrm{h}_{e x}\right)$ on momentum space Berry curvature, we plot the Berry curvature as function of wave number $(k a / \pi)$ in the vicinity of $\mathrm{K}^{+}$and $\mathrm{K}^{-}$valleys for spin-up holes in valence band Figure 2, enhancement of pick of curvature with rise in dopant introduced exchange field (0 to $0.16 \mathrm{eV})$ for state in the vicinity $\mathrm{K}^{+}$valleys are observed Figure 2 .
On the other hand, Berry curvature is related to both spin Hall and valley Hall through relation (20). Therefore, the spin and valley Hall conductivity is expected to increase with dopant introduced exchange field for state in the vicinity of $\mathrm{K}^{+}$valleys. In contrast with Berry curvature of state in the vicinity of $\mathrm{K}^{+}$valley, the Berry curvature of state in the vicinity of $\mathrm{K}^{-}$valley suppresses with rise in dopant introduced exchange field ( 0 to $0.16 \mathrm{eV}$ ). Therefore, the degeneracy of curvature of $\mathrm{K}^{+}$and $\mathrm{K}^{-}$is lifted under dopant introduced magnetic exchange field or these valleys become spin polarized (unequal number of spin-up states are accumulated in $\mathrm{K}^{+}$and $\mathrm{K}^{-}$curvature). In addition to this, at absolute zero $(\mathrm{T}=0 \mathrm{~K})$ and insulating regime where the chemical potential is in gap, (24) and (25) show that the universal value of conductivity $q^{2} / h$ is obtained for single spin and single valleys. Hence, we can say that Hall conductivity is quantized in this limit and the signs of conductivity in $\mathrm{K}^{+}$and 


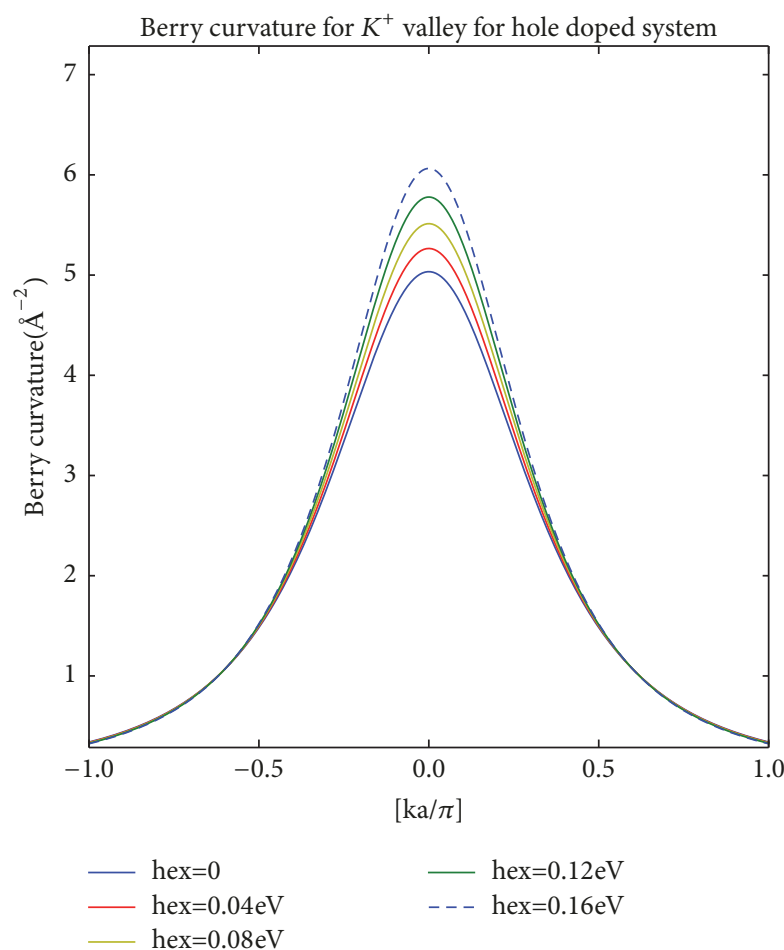

(a)

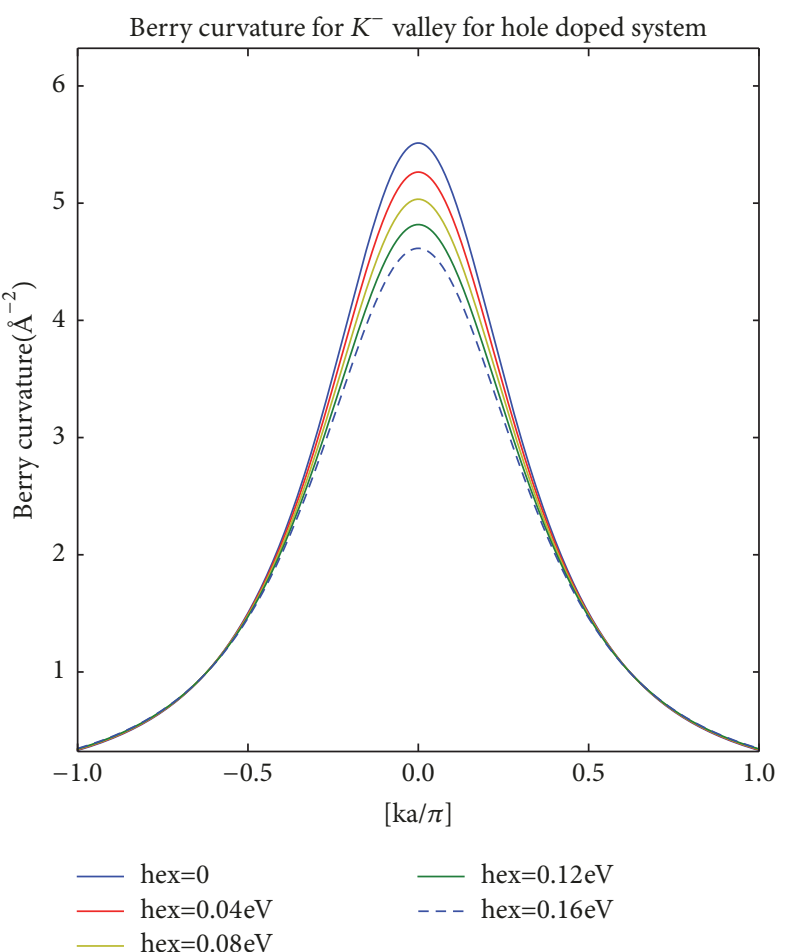

(b)

FiguRE 2: Berry curvature as function of $(k a / \pi)$ for different values of exchange fields $\left(\mathrm{h}_{e x}\right)$, right panel for $\mathrm{K}^{+}$, and left panel for $\mathrm{K}^{-}$valley.

$\mathrm{K}^{-}$are opposite indicating that both of valleys are related to time reversal symmetry. On the other hand, from (26) and (27) and the total spin Hall conductivity vanishes identically. Whereas total valley Hall conductivity is integral multiple of quantum conductivity $\left(4\left(q^{2} / h\right)\right)$ the term 4 appears from twofold valley degrees of freedom (DOF) corresponding to $\mathrm{K}^{-}$and $\mathrm{K}^{+}$valleys of the $\mathrm{BZ}$ and twofold to spin degree of freedom of those carriers. We have also calculated both spin and Valley Hall conductivities at finite doping concentration. The calculated result reveals that in contrast to conductivity in insulating regime Hall conductivity is not quantized but depends on parameters $\left(\Delta_{m}, \lambda_{s o}, h_{e x}, k_{F}\right.$, a, and $\left.\mathrm{t}\right)$; see ((32)(35)). The calculated total spin Hall conductivity and total valley Hall conductivity in (34) and (35) can be written in more compact form as

$$
\begin{aligned}
& \sigma^{s p i n}(z) \\
& =\frac{2 q^{2}}{h}\left\{\left(1+\frac{a^{2} t^{2} k_{F}^{2}}{\left(\Delta_{m}+h_{e x}-(1 / 2) \lambda_{s o}\right)^{2}}\right)^{-1 / 2}\right. \\
& \left.\quad-\left(1+\frac{a^{2} t^{2} k_{F}^{2}}{\left(\Delta_{m}+(1 / 2) \lambda_{s o}-h_{e x}\right)^{2}}\right)^{-1 / 2}\right\} . \\
& \sigma^{\text {val }}(z) \\
& =-\frac{2 q^{2}}{h}\left\{\left(1+\frac{a^{2} t^{2} k_{F}^{2}}{\left(\Delta_{m}+h_{e x}-(1 / 2) \lambda_{s o}\right)^{2}}\right)^{-1 / 2}\right.
\end{aligned}
$$

$$
\left.+\left(1+\frac{a^{2} t^{2} k_{F}^{2}}{\left(\Delta_{m}+(1 / 2) \lambda_{s o}-h_{e x}\right)^{2}}\right)^{-1 / 2}\right\} .
$$

From (36) and (37), total spin Hall conductivity and valley Hall conductivity depend on parameters: $\Delta_{m}, \lambda_{s o}, \mathrm{~h}_{e x}, \mathrm{k}_{F}$, $\mathrm{a}$, and t. For particular limits $\mathrm{h}_{e x}=\lambda_{s o}=0$ and $\mathrm{h}_{e x}=$ $2 \lambda_{s o}$ total spin Hall conductivity in (36) vanishes whereas the total valley Hall conductivity is simplified to $-\left(4 q^{2} /\right.$ h) $\left(\left(\Delta_{m}^{2}+a^{2} t^{2} k_{\mathrm{F}}^{2}\right) / a t k_{F}\right)^{1 / 2}$, which indicates that the interplay between spin-orbit coupling and dopant introduced magnetic exchange field is crucial for existence of spin and valley Hall conductivity.

\section{Conclusion}

In conclusion, based on a three-band tight binding model we have studied valley polarization, spin, and valley Hall conductivity in the presence of dopants introduced magnetic exchange field. The result reveals that in pure limit Hall conductivity in single valley is quantized. In finite doping concentration the existence of dopant introduced exchange field breaks the time inversion symmetry and decouples the energetically degenerated valley into nondegenerate and elucidating the occurrence of valley polarization. We have shown that the Berry curvature of occupied state resulting from transverse motion unequal number of carriers in both valleys of $2 \mathrm{D}$ hexagonal lattice is responsible for existence of 
valley and spin Hall conductivity. Moreover, we have found that, at finite dopant concentration, the strength of the spinorbit coupling together with the exchange energy determines the valley polarization, which in turn controls total valley and spin Hall conductivity in doped monolayer $\mathrm{MoS}_{2}$ system. The spin Hall and valley Hall conductivity are dissipationless in the absence of any external magnetic field. This effect could enable a new generation of low power electronic devices.

\section{Data Availability}

No data were used to support this study.

\section{Conflicts of Interest}

The authors declare that there are no conflicts of interest regarding the publication of this paper. Submitting authors are responsible for coauthors declaring their interest.

\section{Acknowledgments}

This work was supported by the School of Graduate Studies of Addis Ababa University, Addis Ababa, Ethiopia, and Arbaminch University, Arbaminch, Ethiopia.

\section{References}

[1] A. H. Castro Neto, F. Guinea, N. M. R. Peres, K. S. Novoselov, and A. K. Geim, "The electronic properties of graphene," Reviews of Modern Physics, vol. 81, no. 1, pp. 109-162, 2009.

[2] D. Xiao, G. Liu, W. Feng, X. Xu, and W. Yao, "Coupled Spin and Valley Physics in Monolayers of ", Physical Review Letters, vol. 108, no. 19, 2012.

[3] K. F. Mak, K. L. McGill, J. Park, and P. L. McEuen, “The valley hall effect in MoS2 transistors," Science, vol. 344, no. 6191, pp. 1489-1492, 2014.

[4] W. Yao, A. H. MacDonald, and Q. Niu, "Optical Control of Topological Quantum Transport in Semiconductors," Physical Review Letters, vol. 99, no. 4, 2007.

[5] W. Yao, D. Xiao, and Q. Niu, "Valley-dependent optoelectronics from inversion symmetry breaking," Physical Review B: Condensed Matter and Materials Physics, vol. 77, no. 23, 2008.

[6] H. Min, J. E. Hill, N. A. Sinitsyn, B. R. Sahu, L. Kleinman, and A. H. MacDonald, "Intrinsic and Rashba spin-orbit interactions in graphene sheets," Physical Review B: Condensed Matter and Materials Physics, vol. 74, no. 16, 2006.

[7] Y. Yao, F. Ye, X. Qi, S. Zhang, and Z. Fang, "Spin-orbit gap of graphene: First-principles calculations," Physical Review B: Condensed Matter and Materials Physics, vol. 75, no. 4, 2007.

[8] A. Umer, S. Naveed, N. Ramzan, and M. S. Rafique, "Selection of a suitable method for the synthesis of copper nanoparticles," Nanotech, vol. 7, no. 5, Article ID 1230005, 2012.

[9] H. Zeng, J. Dai, W. Yao, D. Xiao, and X. Cui, "Valley polarization in MoS2 monolayers by optical pumping," Nat Nanotechnol, vol. 7, no. 8, pp. 490-493, 2012.

[10] T. Cao, G. Wang, W. Han, and H. Ye, "Valley-selective circular dichroism of monolayer molybdenum disulphide," Nature Communications, vol. 3, article no. 887, 2012.
[11] K. F. Mak, K. He, J. Shan, and T. F. Heinz, "Control of valley polarization in monolayer $\mathrm{MoS}_{2}$ by optical helicity," Nature Nanotechnology, 2012.

[12] G. Kioseoglou and A. T. Hanbicki, "Anomalous temperature dependent spin-valley polarization in monolayer WS2," Scientific Reports, vol. 6, article no. 18885, 2016.

[13] J. Shi, G. Vignale, D. Xiao, and Q. Niu, "Quantum Theory of Orbital Magnetization and Its Generalization to Interacting Systems," Physical Review Letters, vol. 99, no. 19, 2007.

[14] A. Srivastava, M. Sidler, A. V. Allain, and D. S. Lembke, "Valley Zeeman effect in elementary optical excitations of monolayer $\mathrm{WSe}_{2}$," Nature Physics, pp. 141-147, 2015.

[15] A. V. Chumak, V. I. Vasyuchka, A. A. Serga, and B. Hillebrands, "Magnetic control of valley pseudospin in monolayer $\mathrm{WSe}_{2}$ ", Nature Physics, vol. 11, no. 6, pp. 148-152, 2015.

[16] D. MacNeill, C. Heikes, K. F. Mak, and Z. Anderson, "Breaking of Valley Degeneracy by Magnetic Field in Monolayer MoSe $e_{2}$," Physical Review Letters, vol. 114, no. 19, Article ID 037401, 2015.

[17] Y. Li, J. Ludwig, T. Low, A. Chernikov, and X. Cui, "Valley Splitting and Polarization by the Zeeman Effect in Monolayer $\mathrm{MoSe}_{2}$," Physical Review Letters, vol. 113, Article ID 266804, 2014.

[18] A. Kormányos, G. Burkard, M. Gmitra, J. Fabian, and V. Zólyomi, "k.p theory for two-dimensional transition metal dichalcogenide semiconductors," 2D Materials, vol. 2, Article ID 022001, 2015, https://arxiv.org/abs/1410.6666.

[19] S. Mekonnen and P. Singh, "Berry approach to intrinsic anomalous Hall conductivity in dilute magnetic semiconductors (Ga1xMnxAs)," World Journal of Condensed Matter Physics, vol. 5, pp. 179-186, 2015.

[20] Y. Yao, L. Kleinman, A. H. MacDonald, J. Sinova, and T. Jungwirth, "First Principles Calculation of Anomalous Hall Conductivity in Ferromagnetic bcc Fe," Physics Letters B, vol. 92, 2004.

[21] G. Liu, D. Xiao, Y. Yao, X. Xu, and W. Yao, "Electronic structures and theoretical modelling of two-dimensional group-VIB transition metal dichalcogenides," Chemical Society Reviews, vol. 44, no. 9, pp. 2643-2663, 2015. 

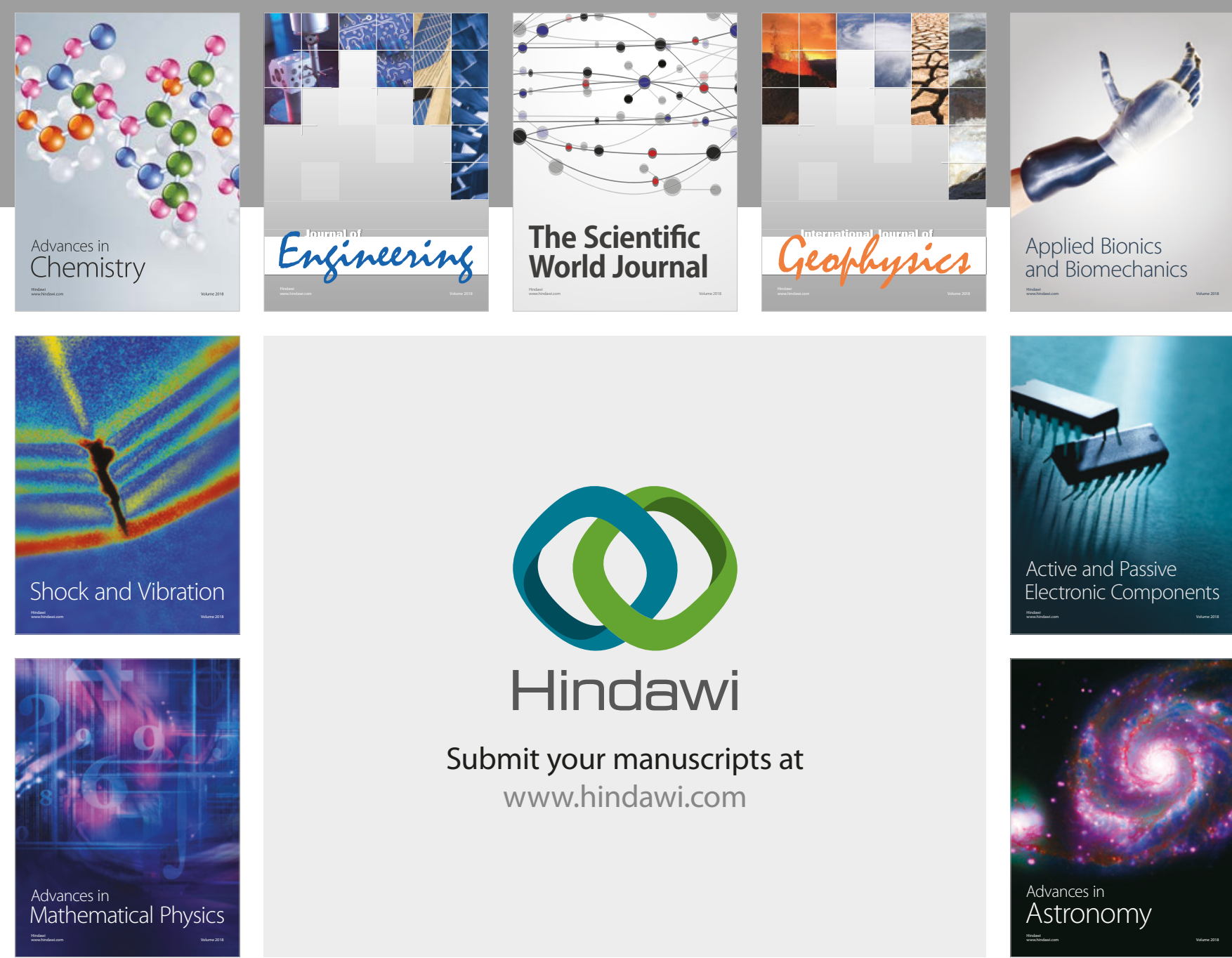

Submit your manuscripts at

www.hindawi.com

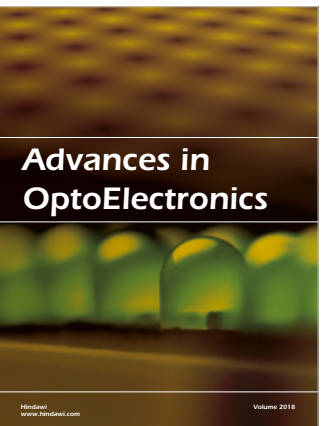

\section{Rotcting Machinery}
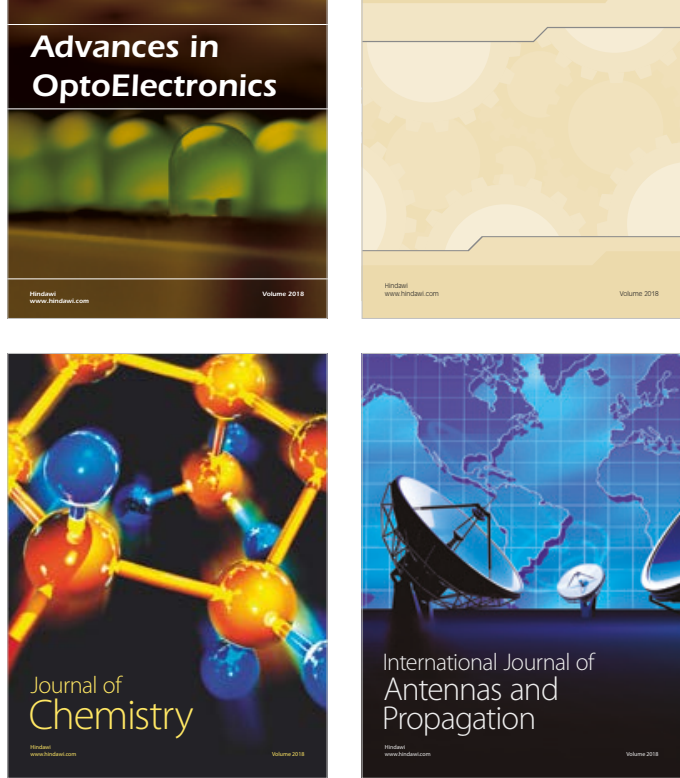

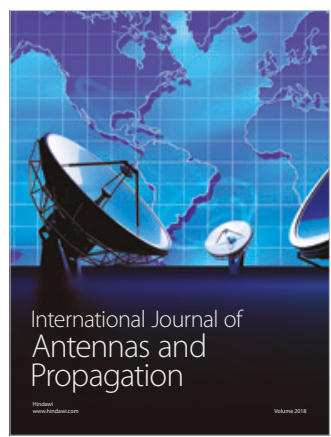

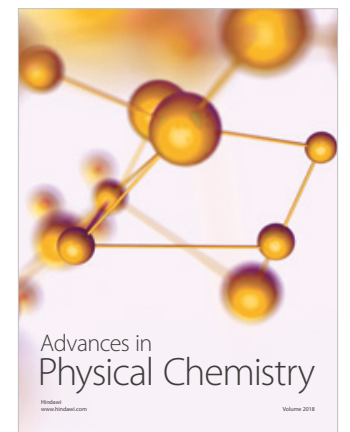

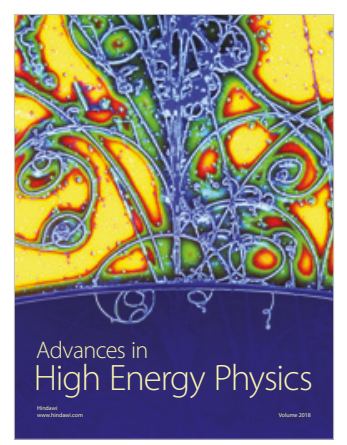

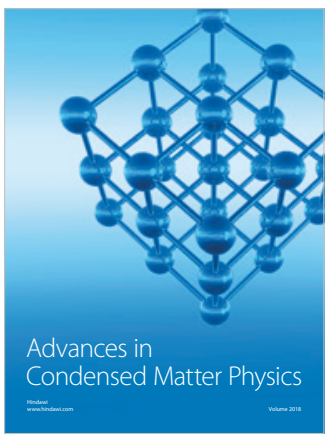

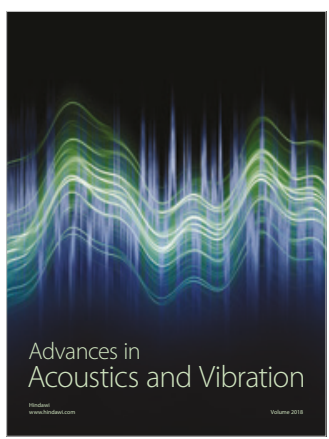

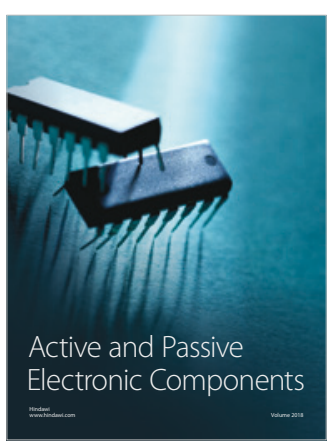
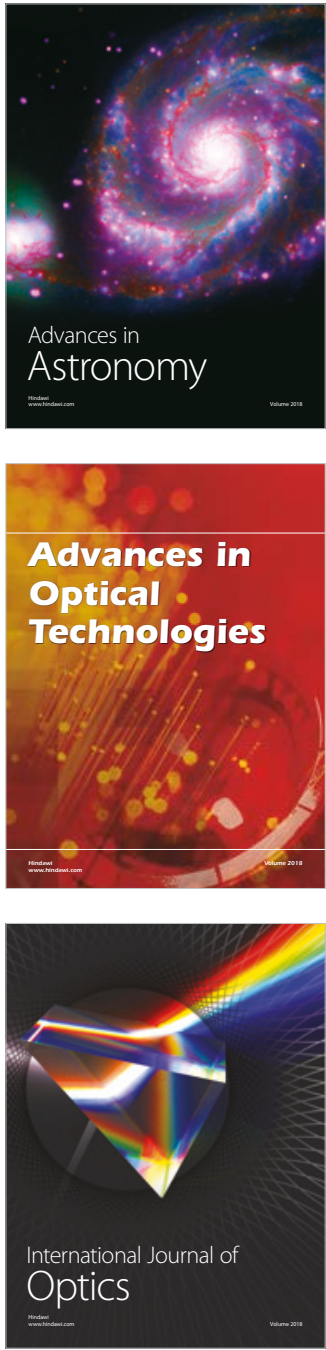